\title{
New Trends in Location-Based Services Technology
}

\author{
Imran Ahmad ${ }^{1}$, Mohammed S BinMelha ${ }^{1,2}$, Mohammed J. Ngala ${ }^{3}$, Raed Abd-Alhameed ${ }^{2,4}$ \\ I.ahmad3@bradford.ac.uk, abofaras@yahoo.com, mjngala@heatoned.co.uk, R.A.A.Abd@bradford.ac.uk \\ ${ }^{1}$ Faculty of engineering and informatics, University of Bradford, Bradford, BD7 1DP, UK \\ ${ }^{2}$ System Maintenance Controller, Saudi Air Navigation Sevices, Saudia \\ ${ }^{3}$ Heaton Education Bradford UK \\ ${ }^{4}$ Information and Communication Eng. Department, Basrah University College of Science and \\ Technology, Basrah 24001, Iraq
}

\begin{abstract}
Because of technological advancements, location-based services are always evolving with the passage of time. The current study discussed significant developments in the field of locationbased services technology. LBS Indoor Systems, LBS Satellite Systems, LBS GPS System, and Outdoor Systems employing Terrestrial BSs are all part of the localization infrastructure. The report also discusses the benefits and drawbacks of various outdoor systems that employ Terrestrial Base Stations, such as the Global System for Mobile Communications. WCDMA (with IPDL, TA-IPDL, and OTDOA-PE), Smart Antenna Systems, and Distributed Antenna Systems, GSM (with E-OTD), GSM (with CELL-ID), CDMA/GPRS (with A-GPS), CDMA/GPRS (with A-GPS), CDMA/GPRS (with A-GPS), CDMA/GPRS (with A-GPS), CDMA/GPRS (with A-GP (with A-GPS) AFLT+AGPS, Wireless Assisted GPS and AFLT: GPS One A Hybrid Solution, WCDMA (with IP), WCDMA (with IP), WCDMA (with IP), WCDMA (with IP), WCDMA (with IP), WCDMA (with IP), WCDMA (DAS). Some of the techniques discussed in this paper are localization by Cell-ID, localization by Prediction, localization by Angle of Arrival (AOA), localization by Time of Arrival (TOA) and Observed Time Difference of Arrival (TDOA), localization by Finger Printing, and Hybrid Localization AOA-TOA Based. Low-power wide-area networks (LPWANs), cellular 5Gbased positioning technologies, ZigBee (Mesh Protocols), and WiFi6 have all been studied in relation to the Internet of Things (IOT). This document also covers NLBS (which includes Bluetooth Low Energy technology, WLAN, and Infrared), UWB, and Vehicle to Vehicle Communications.
\end{abstract}

Keywords: Location-Based Services, LBS, GSM and RFID Systems, GPS, LBS, applications of LBS, AOA, TDOA, UWB

\section{Introduction}

LBSs are a hybrid of commercial and consumer services that rely on the users' placement. Position Level, Tracking Level, and Planning Level are three different degrees of location-based services. The user's current position is required by the Position Level. The Tracking Level requires the user's current and previous positions, whereas the Planning Level requires the user's current, past, and future positions [1]. Itinerant (services provided to a moving user), Distributed (offering the same services to any place within the network), and Ubiquitous (providing the same services to any location inside the network) are further characteristics that define and characterise LBS technology.

\section{Categorization of LBS}

Radio-based systems, ultrasonic-based systems, active infrared-based systems, camera-based systems, and Smart Floor systems are all used in LBS. The disadvantage of radio-based systems is that the objects to be located require particular hardware. Kemper and hybrid systems, such as Active Badge, use ultrasonic-based technologies. The Active Badge System is based on Active Infrared [2]. Camerabased systems have the advantage of being hardware-independent. The Easy-Living-Tracker is an example of this. Wireless networks, user devices, location technologies, geographical data servers, and application servers are all used in LBS [3]. The use of a distributed architecture in LBS can help to reduce network congestion.

There are several methods for determining the positions of items in Location-Based Services, including Terminal-Based Approaches and Network-Based Approaches. Based techniques include the Global Positioning System (GPS), Assisted Global Positioning System (A-GPS), and Enhanced Observed Time Distance (E-OTD). Cell Global Identity (CGI) and Time of Arrival are two examples of 
network-based approaches (TOA). In Location-Based Services, there are various positioning systems for mobile terminals that differ in terms of dependability, precision, and computations. Satellite positioning systems, network-based positioning systems, and local positioning systems are examples of these systems [4]. The reliability, efficiency, and performance of Location Based Services are all influenced by positioning technologies. Indoor, outdoor, or hybrid positioning systems are available. Indoor localization necessitates high precision and signal strength. Flora, Ficco, Russo, and Vecchio (2005) suggested a software architecture that combined interior and outdoor location sensing. The client-side mechanism and zoning location sensing approach were used to create this architecture. It complies with JSR-179 requirements [5].

LBS Indoor Systems, LBS Satellite Systems, and LBS Outdoor Systems Using Terrestrial BSs are all part of the localization infrastructure. Localization accuracy, availability, and consistency, applicability, power consumption, hardware size, software size, processing load, supported positioning modes, network-dependent and signalling load, cost, standardisation, and delay are all quality criteria for localization technologies. The advantages, disadvantages, and applications of different Outdoor System Localization are discussed below:

\subsection{Radio Frequency Identification (RFID)}

The RFID beacons are small and light. It works by sending out little radio packets at short wavelengths. RFID employs biometrics, voice recognition, smart cards, optical character recognition, bar codes, and other forms of automatic identification. It collects data about a specific item via electromagnetic waves or inductive coupling [6]. RFID advancements have resulted in various benefits, including resource optimization, increased corporate transaction efficiency, and improved health-care systems. RFID is utilised in manufacturing for resource management, quality control management, inventory management, and tracking management [7]. RFID is utilised for shipping management, picking management, and receiving management in warehousing [8]. In the transportation industry, it's utilised to control safety, distribution, and material processing. The truck drivers, trucks, and the company to which the trucks belong are all verified using RF tags on the trucks. The Commercial Driver's License with the tags validates that they are visiting the terminals for business. The contents of the containers on the trucks can also be determined using this system [9]. For checkout management, shelf-stock management, and inventory management in the retail industry. RFID inventory management enhances merchandise onshelf availability and delivers applications like smart fitting rooms, smart checkout, and product location tools [10]. It can be used for crop identification, animal tracking, and animal diagnostics in agriculture. This technology is employed throughout the seed quality management process, including seed cultivation, storage, and transportation to the supervision department, seed quality confirmation, and seed distribution to farmers [11]. It can be utilised for public transportation tickets, automatic vehicle locating, toll collection, and smart car keys in ticketing and traffic control. It is utilised in pre-paid access cards with an incorporated microchip for toll payment. An Automated Parking Management System employs RFID technology. The Integrated Recognition and Identification System (IRIS) [12] connects Automatic Number Plate Recognition (ANPR) technology with RFID technology in this system. RFID can be employed in a variety of applications, including aged care, pharmaceutics, hospital equipment and personnel management, implants, prosthesis, and patient medical history tracing. To combat the problem of counterfeit medications in the United States, the Food and Drug Administration (FDA) has mandated the use of RFID pedigrees on all wholesale prescription drug shipments [13]. In Finance, it can be used in smart cars and currency identification. The American Express launched a credit card in 2005, which provided the facility to the customers to purchase items without swiping their cards [14]. It can be used for digital identification, electronic passports, facility access, and punishment system management. The rate of crime can be minimised by adopting RFID technology for human identification management because there will be less risks of fraud or theft.

In sports and games management, it can be used for gaming chips, sports event timing and tracking golf balls [15]. The Washington Major League Football team has made the football game more interesting by employing RFID technology in tickets, purchasing of food and drinks at refresher stands. This system has also been introduced at Nationals' Park. Fortress GB, which is a British sports and entertainment technology is deploying RFID enabled card for ticketing systems [16]. In clothing, it can be used for laundry ID and cleaning control system management. RFID tags are also useful for the garment business. RFID tags provide enough space for data storage for a wide range of information and applications. Miniature RFID tags are utilised in teaching and research. For example, in 2009, Bristol 
University researchers used RFID transponders to analyse ant behaviour [17]. As a result, RFID technology is very beneficial for studying animal and bird life patterns, habitats, and habits.

\subsection{Global Positioning Systems (GPS)}

The GPS is a functioning system that includes an earth-orbiting satellite-based routing system [18]. The Global Positioning System is used by military units, sailors, utility companies, evaluators, pilots, and hikers with small hand-held receivers. GPS satellites have feeble transmissions that cannot pass the walls of buildings, therefore it does not perform well in an inside environment. GPS technology is employed in a wide range of applications. The following sections go over some of the applications:

Satellite navigation in ships, aircraft, and cars is the most common use of GPS. It allows a GPS receiver to pinpoint their location or speed with pinpoint accuracy. It not only gives cars with a path to follow, but it also delivers traffic alerts. In emergency scenarios, GPS can be quite useful. It aids hikers in staying on track and arriving at their intended destination [19].

GPS is inexpensive and so accessible to the general public. It provides unrestricted access all over the world [20]. GPS delivers high-resolution data for mapping and surveying. When compared to other mapping and surveying approaches, the data collection is far too fast - less than a single day. The data collected from GPS can be displayed on maps that can be stored or manipulated. One of the most important applications of GPS regarding surveying is that it is not restricted to the sight visibility between survey stations [21]. In global financial systems, millions of money transfers and transactions whether local or international takes place every day. GPS can provide accurate dates and times for electronic funds transfer, ultimately serving a critical purpose for financial systems worldwide. GPS is used in engineering applications such as construction, mining and guiding heavy vehicles. It allows the driver to follow a surveyors' preprogrammed site plans to reach for the nearest accuracy and efficiency in work [22].

Glimpse is a mobile location sharing programme that allows users to broadcast their position via text messaging and social media. This app also provides route information, traffic alerts, and alternate routes. By employing their cell phone's application, GPS track pro may simply locate anyone [23]. The jamming properties of GPS have a considerable application in military navigation. The positioning of forces is critical in military processes and operations. It assists in the tracking and monitoring of possible targets [24]. Other techniques, such as photo-reconnaissance, can be used in these tracking procedures to improve awareness and information about bombing missions. Unmanned Aerial Vehicles (UAVs) are used in military operations all over the world in the current day. GPS Jammer Location (JLOC) [25] is one of the military applications. It's made to keep track of GPS interference threats and send out alerts if any are identified in the region.

Assisted GPS is ubiquitous in today's GPS systems, yet few people are aware of it. The satellite system and an external server are used by A-GPS [26]. The most significant benefit of A-GPS is that the signals are always whole and contain no fragments. Second, it is speedier because it makes advantage of the nearby cell towers, which provide a clear picture of the location. A subscription is necessary to utilise AGPS, which is pricey. Because most A-GPS devices are reliant on an external server, they may not function effectively without it [27].

\subsection{Outdoor system using Terrestrial BSs}

The Outdoor system employing Terrestrial includes GSM (with E-OTD), GSM (with CELL-ID), CDMA/GPRS (with A-GPS), CDMA/GPRS (with A-GPS) - AFLT+AGPS, Wireless Assisted GPS and AFLT: GPS One A Hybrid Solution, WCDMA (with IPDL, TA-IPDL, and OTDOA-PE), Systems with Smart Antennas, and Distributed Antenna (DAS). The advantages and disadvantages of these systems are discussed below:

\subsubsection{Global System for Mobile Communication (GSM)}

The modern cellular technology used to transmit voice and data around the world is known as the GSMC. Users of GSM phones can travel to more than 218 countries with the same number. Different algorithms can be used by GSM users to obtain location fixes [28]. GSM has a number of drawbacks, including bandwidth slowness and a proclivity to interact with certain electronic devices. Its pulse transmission method can interfere with a pacemaker or airport radio communications. 


\subsubsection{GSM (with E-OTD)}

GSM mobile operators employ E-OTD as a multi-lateration positioning approach to find its users [29]. It's similar to the TDA technique, except that algorithm and all other calculations are done in the user's device or handset instead of the base station or server. A lot of data is needed to make the equations easier to understand, including different time measurements, which are referred to as Location Measurement Units (LMU) [30]. Installing Location measurement equipment throughout the coverage area, as well as equipping the user's handset with appropriate software, is both costly.

\subsubsection{GSM (with Cell- ID)}

Network providers is assigned base transceiver stations to ensure full coverage. The regions were then further separated, with each receiving a Cell ID and BS to to connect with users. As a fix to the Cell ID would limit the position to some amount, GSM providers might now utilise multiple localization techniques to place a user [31].

\subsubsection{CDMA/GPRS (with A-GPS)}

The GSM GPRS tracker is one of popular location trackers on the market. The CDMA GPS trackers differ from the GPRS GPS trackers in that they use a GPS-One chipset, which is less accurate. Assisted GPS is used by the CDMA tracker. To locate an object, A-GPS uses fragmented data stored on a network server [32]. The CDMA tracker is substantially expensive to GPRS since it requires additional server storage.

\subsubsection{CDMA/GPRS (with A-GPS) - AFLT+AGPS}

When it comes to outdoor positioning, the Hybrid GPS and AFL Trilateration is the best and most accurate. Even when a GPS-only solution is unavailable, our hybrid approach gives the most precise position. There is a tiny drawback in acknowledging that if a GPS-only solution is not accessible, and a direct LoS of trilateration requires three BSs and there is a danger of inaccuracy or ambiguous placement [33].

\subsubsection{Wireless Hybrid Assisted GPS and AFLT}

Mixture localization (hybrid solution) combines the best of both worlds. The cellular network and GPS are used by GPS-One. It improves GPS receiver sensitivity and delivers accurate localizations even when less than four satellites are visible [34]. The only drawback is that it comes with its own hardware and software, as well as the need for an installed chipset.

\subsubsection{Systems with Smart Antenna}

Adaptive array antennas are used in Smart Antennas to improve the efficiency of wireless communications networks. Signal processing algorithms can be used for tracking user with new arrangement of array antennas [35]. Using smart antennas provides a variety of advantages, including the ability to cater to a considerably many users at the same time. Furthermore, because of their directionality, they provide greater security and less interference than traditional Omni-directional antennas.

\subsubsection{Distributed Antenna Systems (DAS)}

A DAS is a system that uses coaxial wire, fibre optic cable, and antennas to boost cellular, public safety, and other communications inside a building [36]. The DAS system is crucial in radar applications, such as vehicle-mounted surveillance radars and radar system such as anti-missile with restricted energy sources [37]. DAS improves wireless coverage, data speed, and call clarity in these circumstances by eliminating poor wireless reception. In a Neutral Host DAS concept, the expense of providing services based on sharing the number of carriers, allowing low and high data rate venues to become economically viable [38].

\section{Techniques for the Existing Localization}

Some of the existing localization approaches are Cell-ID, Prediction (Dead-Reckoning Method), Localization by Angle of Arrival (AOA), Localization by Time of Arrival (TOA) and Observed Time Difference of Arrival (OTD), Hybrid Localization AOA-TOA Based, and Localization by Finger Printing. The advantages and disadvantages of these technologies are discussed below:

\subsubsection{Localization by Cell-ID}


To locate a mobile user, position of Cell ID needs the existing cellular network address. Covering multiple cells and smaller regions the base transceiver station communicates ware responsible to provide that bases. The BTS connects with the user's device via a distinct signal transmitter and Cell ID for each cell. The network examines the user Cell ID who has requested localisation while positioning.

\subsubsection{Localization by Dead -Reckoning}

While navigating, Dead Reckoning is a mechanism for calculating position of user from previously calculated positions and forwarding it based on predicted motion. Advanced satellite systems have sealed the market for dead reckoning in the world of navigation; [40] however, inertial navigation systems that give precise information about direction, use Dead Reckoning efficiently and are very widely applied.

\subsubsection{Localization by AOA}

After determining the angle at which signals are received at numerous receivers on a base station, localization by Angle of Arrival is performed by computing node locations using fundamental geometry formulae. Angle of Arrival (AOA) approaches, in general, produce more accurate localization results than Received Signal Strength Indicator (RSSI)-based techniques, however the hardware cost of this technology is relatively high. Localization by AoA is particularly inaccurate and time-consuming for a rapidly moving item since the many receivers at the BS do not account for the time gap [41].

\subsubsection{Localization by TOA and Observed TDOA}

Localization by TOA is an approach for calculating a mobile user's location [42]. When user in LoS this technique is quite accurate. However, there is a problem that the wave progression in air varies depending on environmental variables such as temperature and humidity. This causes error in distance estimation-based localisation [43].

Localization by Observed TDoA, is a multi-iteration technique. In this technique, the system localizes a user by using time-synchronized receivers. Localization is done by multiple nodes of known locations that report the relative time measurements of receiving a signal, rather than the absolute time.

\subsubsection{Hybrid Localization AOA-TOA}

Hybrid reference are a combination of several single localization approaches that solve the problems, such as TOA, AOA, and TDoA, have when used alone. LoS between the receiving beacon and the mobile source is resolved using a hybrid of Time of Arrival and Angle of Arrival. Because Angle of Arrival can't tell the difference between direct and reflected signals, the system uses a variety of efficient techniques to get around issue. The advantages are clarity, accuracy, and dependability. One of the drawbacks of the Hybrid system is the increased computational cost, as more calculations are at hand now. To speed up the localisation, new and far more complicated algorithms are applied [44]. Furthermore, specialised hardware may be more durable and costly [45]. Both ends should be time-synchronized for TOA-AOA to work.

\subsubsection{Localization by Finger Printing}

The term "fingerprinting" refers to a method of recognising a certain location based on the data that represents it. Fingerprinting approaches are particularly successful at identifying a mobile user in an indoor setting, as well as in complex problem the traditional localization techniques based on ToA or triangulation are promoted but with major inaccuracies, such as canyons and tunnels [46]. The fingerprinting database must be sized big to obtain more accuracy. Furthermore, the database must be updated every time the environment changes. Fingerprinting is very precise and accurate but very expensive to run and maintain in the outdoors.

\section{Internet of Things (IOT)}

It comprises of a system of interconnected computing devices, mechanical as well as digital machines, animals, objects, or humans that are supplied with unique identifiers and the capability of communicating without needing human-to-human interaction or human to computer interaction [47]. Examples of Internet of Things include sensors for monitoring traffic conditions, heart sensors for cardiac patients, security and home management, moisture sensors for directing irrigation, location sensors for cattle, sensors for monitoring hazardous material in industries, sensors for managing employees' working conditions, automated inventory control, etc. The Internet of Things comprises of leading technologies such as Low Power Wise Area Network (LPWANs), Cellular 5G based positioning methods, ZigBee (Mesh Protocols) and WiFi6. 


\subsection{Low Power Wise Area Network (LPWANs)}

It is the novel technology in Internet of Things that supports large-scale Internet of Things networks ranging over wide industrial and commercial sites [48]. Low Power Wise Area Networks can link all kinds of Internet of Things sensors, such as assisting several applications from asset tracking, monitoring of environment, facility management, consumables monitoring, occupancy detection, etc. The drawback of LPWANs is that it can only transmit small blocks of data at a low rate, and hence is appropriate for cases that do not need high bandwidth and are not time-bound.

\subsection{ZigBee (Mesh Protocols)}

Zigbee is a low-power, short-range, wireless standard (IEEE 802.15.4) that is usually implemented in mesh topology for extending coverage by transmitting sensor data over numerous sensor nodes. In comparison to Low Power Wise Area Network, Zigbee gives high data rates, but simultaneously, less power efficiency due to mesh configuration [50]. ZigBee are appropriate for medium-range Internet of Things applications with a uniform allocation of nodes in adjacent propinquity. Generally, Zigbee is an ideal addition to Wi-Fi for different home automation usages such as HVAC controls, security management, energy management, smart lighting, etc.

\subsection{WiFi6}

WiFi 6 is the novel Wi-Fi generation that comprises of improved network bandwidth (that is; $<9.6 \mathrm{Gbps}$ ) for enhancing data per user in over-crowded places [51]. The use of WiFi 6 can raise public Wi-Fi infrastructure and transforms the experience of the consumers with novel digital mobile services, such as, retail and entertainment segments. Moreover, it can be used in car networks for the purpose of infotainment and On-board Diagnostics (Vehicles' self-diagnostic reporting).

\section{Near Location Based Services (NLBS)}

NLBS are a collection of wireless technologies [52]. This programme allows users to get information based on their current location. It's especially well-suited to use in confined places, small spaces, or restricted areas. Usual ranges for other technologies are included.

\subsection{Bluetooth Low Energy (BLE) Technology}

BLE has been initiated to handle small-scale consumer applications related to the Internet of Things, it enabled devices are mainly utilized in combination with electronic devices, usually the smart phones that act as a hub for transmitting data to the cloud network [53]. In the modern era, Bluetooth Low Energy is extensively incorporated in fitness as well as medical wearable (such as glucose meters, smart watches and pulse oximeters) and Smart Home devices (for instance, door locks).

\subsection{Wireless Local Area Network (WLAN)}

WLAN is a network that allows devices to connect and communicate wirelessly [54]. The Dynamic Host Configuration Protocol is commonly used to add and configure new devices (DHCP). They can communicate with other devices on the network in the same way that a wired network would. It enables homeowners and businesses to set up local networks without the need for Ethernet wiring.

\subsection{Infrared}

Infrared (IR) technology, often known as infrared light, is a type of Electromagnetic Radiation (EMR) with visible light wavelengths. Infrared wavelengths are typically defined as spanning from 700 $\mathrm{nm}(\mathrm{f}=430 \mathrm{THz})$ to $1 \mathrm{~mm}(\mathrm{f}=300 \mathrm{GHz})$ in the visible spectrum [55]. Nearly all infrared wavelengths are released by black radiation that are near to room temperature. Infrared broadcasts energy and momentum and has the attributes of both a wave and a particle, the photon, as a type of electromagnetic radiation. When molecules change their vibrational rotational motion, they absorb or emit infrared radiation [56]. It induces vibrational modes in a molecule by changing the dipole moment for studying the energy levels. Infrared spectroscopy studies the absorption and emission of photons in the infrared region. Humans or animals can be viewed without revealing the observer using night vision systems that use active nearinfrared illumination [57]. Sensor-equipped telescopes are used in infrared astronomy to penetrate dusty parts of space, and flow of blood within the skin. 


\section{Ultra Wideband (UWB)}

UWB is a radio technology that can communicate over a large area of the radio spectrum while using low energy. In non-cooperative radar imaging, Ultra Wideband has a traditional implementation [58]. The current applications are focused on collected data by sensors, localised precision, and tracking. UWB [59] is a method for broadcasting information over a large BW $>500 \mathrm{MHz}$. Because of its use in Synthetic Aperture Radar (SAR) technology, Ultra Wideband has received a lot of interest [60]. UWB PulseDoppler radars are also utilized for the monitoring of significant signs inside the body of humans, for instance, heart rate, baby monitoring, human gait analysis, respiration signals, fall detection, etc.

\section{Vehicle to Vehicle (V2V)}

$\mathrm{V} 2 \mathrm{~V}$ communication facilitates vehicles to wirelessly exchange information relevant to their location, speed and direction. The technology that is used in $\mathrm{V} 2 \mathrm{~V}$ communication permits the vehicles up to 10 times/sec messages thus establishing a 360 degree "awareness" of the other vehicles in propinquity [61]. Vehicles that have appropriate software (or safety applications) can utilize the messages from surrounding vehicles for determining the possible crash threats that they can face [62]. The V2V communication messages possess a range of above 300 meters and have the ability of detecting the dangers that have been concealed by weather, terrain, or traffic [63]. V2V communication extends and improves the current accessible crash avoidance systems that utilize radars and cameras for the detection of collision threats. V2V communication system can enhance the performance of vehicle safety systems and can assist in saving lives. The linked vehicle technologies can provide drivers with the tools they require for anticipating possible crashes and considerably reducing the number of lives that are lost yearly.

\section{Conclusion}

Location-Based Services' indoor and outdoor localization strategies have a number of advantages and disadvantages. RFID advancements have resulted in resource optimization, higher corporate transaction efficiency, improved health-care systems, and improved customer service. The disadvantage is that programming RFID for individual applications takes a long time. In comparison to other navigation systems, the GPS system is particularly cost-effective, and the receiver is unaffected by weather or climatic changes. The disadvantage is that it does not perform well in an indoor environment because GPS satellites have feeble signals that cannot penetrate building walls. GSM technology enables global communication and coverage, although there is a BW latency. Smart Antennas have the benefit of simultaneously connecting a higher number of users, re-usable frequencies, various pathways, increased BW, and cost-effective; nevertheless, managing their vast size and position necessitates a lot of planning. DAS minimises shadowing and penetration data losses, but it comes with a significant infrastructure cost and is difficult to maintain. Cell-ID localization is low-power, however it does not cover a large area. Path integration is used in the Dead Reckoning technique of localization, although it has the drawback of error accumulation because the location is predicated on the previously determined position. AOA approaches provide more exact localization results, but they come at a considerable expense in terms of technology.

TOA might use a variety of signals, including RF, infrared, ultrasonic, and so on, but it has the downside of requiring the user to be in LoS of the received signal beacons. Observed TDOA uses timesynchronized receivers to locate a user. This technology is less expensive and quicker, but it requires LoS. Localization Hybrid is a fusion of several single localization approaches of AOA and TOA. Its disadvantage is that it has a higher computational cost. Fingerprinting-based location is particularly effective for indoor and complex environments. It is precise and accurate, but the database must be updated every time the environment changes, which is time consuming and expensive. In the modern era, new technologies have been introduced such as the Internet of Things (IOT) which involves Power Wise Area Network (LPWANs), Cellular 5G based positioning methods, ZigBee (Mesh Protocols) and WiFi6. Moreover, NLBS (involving Bluetooth Low Energy technology, WLAN and Infrared), UWB and Vehicle to Vehicle Communications have improved the localization techniques. Researchers have offered a number of methods for improving the technology and services that are now available for LBSs. LBSs have a bright future into our smart communities.

\section{Acknowledgments}


The European Union's Horizon 2020 research and innovation programme under grant agreement H2020MSCA-ITN-2016 SECRET-722424 and the UK's Research Council (EPSRC) under grant EP/E022936 partially sponsored this work.

\section{Reference:}

1. Abulleif, T. and Al-Dossary, A., Location Based Services (LBS), Surveying Services Division, Saudi Aramco, Dhahran, Saudi Arabia, 2008.

2. Want, R., Hopper, A., Falcao, V., and Gibbons, J., The Active Badge Location System, Olivetti Research Ltd. (ORL), Tech. Rep., 1992.

3. Adusei, I. K., and Kyamakys, F. E. Location Based Services: Advances and Challenges, Master's Dissertation, Institute of Communication Engineering, 2005, University of Hannover.

4. Tsalgatidou, A., et al., Mobile E-Commerce and Location-Based Services: Technology and Requirements, 2003, The University of Athens.

5. Flora, C., Ficco, M., Russo, S., and Vecchio, V., Indoor and Outdoor Location Based Services for Portable Wireless Devices, Proc. $25^{\text {th }}$ International Conference on Distributed Computing Systems Workshop (ICDCSW'05), 2005.

6. S.A. Weis, RFID (Radio Frequency Identification): Principles and Applications, Master's Dissertation, CSAIL, 2007.

7. Ilie-Zudor, E., Kemen, Z., Egr, P., and Monostori, L., The RFID Technology and its Current Applications, Proc. The Modern Information Technology in the Innovation Processes of the Industrial Enterprises- MITIP, 2006: p. 29-36.

8. Shutzberg, L., Radio Frequency Identification (RFID) In the Consumer Goods Supply Chain, RockTenn Company.

9. Nguyen, T. Port trucks get RFID, Fleet Owner, 2006.

10. ZIH Corp. Zebra, Traceability in Retail: Reducing RFID Media Costs for Best Value, 2013.

11. Li, H. Application of RFID in Agricultural Seed Quality Tracking System, Proc. $8^{\text {th }}$ World Congress on Intelligent Control and Automation (WCICA), IEEE, Jinan, China, 2010: p. 3073-3077.

12. Gifford, J., RFID Application in Transportation Operations, George Mason University, San Diego, 2006.

13. United States Department of Health and Human Services, Combating Counterfeit Drugs, Food and Drug Administration, Technical Report, 2004.

14. Swedberg, C., MEX Adds RFID to Blue Credit Cards, RFID Journal, 2005.

15. Smith, A., Balakrishnan, H., Goraczko, M., and Priyantha, N., Tracking Moving Devices with the Cricket Location System, Proc. 2nd International Conference on Mobile Systems, Applications and Services (Mobisys 2004), ACM, Boston, MA, USA, June 2004: p. 190-202.

16. Swedberg, C., RFID is the Ticket at Washington Nationals' Park, RFID Journal, 2012.

17. TFOT, Hitachi Develops World's Smallest RFID Chips, 2007.

18. Jechlitschek, C. A Survey Paper on Radio Frequency Identification (RFID) Trends, 2006.

19. Ergen, et al., Application of GPS to Mobile IP and Routing in Wireless Networks, University of California Berkeley, 2002.

20. MiTAC International Corporation, Applications Of GPS, Available on: www.mio.com/technologyapplications-of-gps.htm

21. Griffin, R. T., Top Benefits Of Using A Handheld, Walking, Or Hiking GPS, Ezine Articles, 2011.

22. Aughey, R. J., Applications Of GPS Technologies To Field Sports, International Journal Of Sports Physiology And Performance, 2011: p. 295-310.

23. Maddison, R. and Clion, N.M. Global Positioning System: A New Opportunity In Physical Activity Measurement, International Journal of Behavioral Nutrition and Physical Activity, 2009.

24. National Coordination Office for Spaced-Based Positioning, GPS: Surveying and Mapping Applications, Navigation and Timing, 2016.

25. Locata Corporation Private Limited, Civilian Applications Of GPS, Australia, 2016.

26. Patil, B., Patil, R., and Pittet, A. Energy Saving Techniques for GPS Based Tracking Applications, Proc. Integrated Communications, Navigation and Surveillance Conference (ICNS), 2011: p. 8-10.

27. Yongwei, M. A Study of Localization Methods on Mobile Platform and WIFI-Based User Movement Detection, University of Delaware. UMI. ProQuest LLC, 2015.

28. Walia, V., and Kaur, S. Survey of Different Localization Techniques of Wireless Sensor Networks, IJEEE, 2014. 
29. Oscar, R., Martin, E. I., and Francisco, A., A Field Study on Satellite and Cellular Signals as Sources for Location, Proc. $2^{\text {nd }}$ International Conference on Computer Engineering and Applications, Bali Island, Indonesia, 2010: p. 474-478.

30. Charitanetra, S. and Noppanakeepong, S. Mobile Positioning Location using E-OTD Method for GSM Network, Proc. Student Conf. Research and Development, 2003: p. 319 -324.

31. Nazarpour, F. A., New Method for Cell Id Assignment for Distance Based Location Management in Cellular Communication Systems, Proc. 3rd International Conference on Information and Communication Technologies: From Theory to Applications (ICTTA), 2008: p. 1-5.

32. Patil, B., Patil, R., and Pittet, A., Energy Saving Techniques for GPS Based Tracking Applications, Proc. Integrated Communications, Navigation and Surveillance Conference (ICNS), 2011.

33. Chia-Yenand, S., and Marron, P. J, COLA: Complexity-Reduced Trilateration Approach for 3D Localization in Wireless Sensor Networks, Proc. $4^{\text {th }}$ International Conference on Sensor Technologies and Applications (SENSORCOMM), 2010: p. 24-32.

34. Soliman, S., et al., GPSOne: A Hybrid Position Location System, Proc. $6^{\text {th }}$ International Symposium on Spread Specturm Techniques and Applications (ISSSTA), IEEE, 2000: p. 1.

35. Zhu, X., Wang, Y., and Zhu, H., A Precise 2-D Wireless Localization Technique Using Smart Antenna, Proc. International Conference on Cyber-Enabled Distributed Computing and Knowledge Discovery, CyberC, 2010: p. 59-63

36. Hoglund, D. H. Distributed Antenna Systems for Healthcare, IT horizons. Inc., 2010.

37. Godrich, H., Petropulu, H. and Poor, H. V., Resource Allocation Schemes for Target Localization in Distributed Multiple Radar Architectures, Procs. 18th European Signal Processing Conference, 2010.

38. Dahrouj, H., and Yu, W., Coordinated Beamforming for the Multicell Muti-Antenna Wireless System, IEEE Transactions on Wireless Communications, 2010. 9(5): p. 1784-1759.

39. Trevisani, E. and Vitaletti, A. Cell-id Location Technique, Limits and Benefits: An Experimental Study, Proc. $6^{\text {th }}$ International Workshop on Mobile Computing Systems and Applications (WMCSA), IEEE, Dec 2004: p. 51-60.

40. Samuel, H., et al., Indoor localization Using Pedestrian Dead Reckoning Updated with RFID-based Fiducials, Proc. $333^{\text {rd }}$ Annual International Conference on Engineering in Medicine and Biology Society, IEEE, 2011: p. 7598-7601.

41. Mesmoudi, A., Feham, M., and Labraoui, N., Wireless Sensor Networks Localization Algorithms: A Comprehensive Survey, International Journal of Computer Networks and Communications (IJCNC), 2013.

42. Zhang, D., Xia, F., Yang, Z., Yao L., and Zhao, W., Localization Technologies for Indoor Human Tracking, Proc. 5th International Conference on Future Information Technology (FutureTech 10), May 2010.

43. Walia, V., and Kaur, S., Survey of Different Localization Techniques of Wireless Sensor Networks, IJEEE, 2014.

44. Khan, M. W., Salman, N., and Kemp, A. H., Enhanced Hybrid Positioning in Wireless Networks 1: AoA-ToA, Proc. International Conference on Telecommunications and Multimedia, TEMU, 2014.

45. Gantea, A. D., and Sillera, M., A Survey of Hybrid Schemes for Location Estimation in Wireless Sensor Networks, Proc. Iberoamerican Conference on Electronics Engineering and Computer Science. Procedia Technology, 2013: p. $377-383$.

46. Yu, L., Laaraiedh, M., Avrillon, S., and Uguen, B., Fingerprinting Localization Based on Neural Networks and Ultra-Wideband Signals, Proc. International Symposium on Signal Processing and Information Technology (ISSPIT), IEEE, Bilbao, Spain, 2011: p. 184 -189.

47. Mattern, F., and Floerkemeier, C., (2010). From the Internet of Computer to the Internet of Things, Informatik-Spektrum. 2010. p. 107-121.

48. Beser, N. B., Operating Cable Modems in a Low Power Mode, U.S. Patent No. 7,2008: p.389,528. 49. Rappaport, T. S., et al., Millimeter Wave Mobile Communications for $5 G$ Cellular: It Will Work, IEEE Access, 2013. p. 335-349.

50. Bellido-Outeirino, F. J., Building Lighting Automation through the Integration of DALI with Wireless Sensor Networks, IEEE Transactions on Consumer Electronics, 2012. 58 (1): p. 47-52.

51. Khorov, E., Kiryanov, A., Lyakhov, A., and Bianchi, G., A Tutorial on IEEE 802.11ax High Efficiency WLANs. IEEE Communications Surveys \& Tutorials. IEEE. 2019. p. 197-216.

52. Martin, E.., Vinyals, O., Friedland, G., and Bajcsy, R., Precise Indoor Localization Using Smart Phones, ACM Multimedia, 2010: p. 787-790.

53. Honkanen, M., Lappetelainen, A., and Kivekas, K., Low end extension for Bluetooth. 2004 IEEE Radio and Wireless Conference 19-22 September 2004. IEEE. 2004: p. 199-202.

54. Al Shourbaji, I., An Overview of Wireless Local Area Networks, Computer Networks Department, Jazan University. Jazan, Saudi Arabia, 2005. 
55. Rowan-Robinson, M., Night Vision: Exploring the Infrared Universe, 2013: Cambridge University Press, p. 23.

56. Haynes, William M., (2011). CRC Handbook of Chemistry and Physics (92 ${ }^{\text {nd }}$ ed.), 2011: CRC Press, p. 10.233 .

57. Lynch, David K.; Livingston, William Charles (2001). Color and Light in Nature (2 ${ }^{\text {nd }}$ ed.), 2001: Cambridge, UK: Cambridge University Press, p. 231.

58. Zhou, Y., Law, C. L., Xia, J. (2012). Ultra low-power UWB-RFID System for Precise Location Aware Applications, IEEE Wireless Communications and Networking Conference Workshops (WCNCW), 2012: p. 154-158.

59. Kshetrimayum, R., An Introduction to UWB communication Systems, IEEE Potentials, 2009. p. 913.

60. Ren, L., Wang, H., Naishadham, K., Kilic, O., and Fathy, A., Phase-Based Methods for Heart Rate Detection Using UWB Impulse Doppler Radar, IEEE Transactions on Microwave Theory and Techniques, 2016. p. 3319-3331.

61. Boehmlaender, D., et. al., Advantages in Crash Severity Prediction Using Vehicle to Vehicle Communication, 2015 IEEE International Conference on Dependable Systems and Networks Workshops. IEEE, 2015: p. 112-117.

62. Harding, J., Vehicle to Vehicle Communications: Readiness of V2V Technology for Application, 2014: U.S Department of Transportation, NHTSA.

63. Zorkany, M., Yasser, A., and Galal, A. I., Vehicle To Vehicle "V2V” Communication: Scope, Importance, Challenges, Research Directions and Future, The Open Transportation Journal, 2021. 14(1): p. 86-98. 